\title{
La calidad de vida urbana y la dimensión físico espacial del espacio público: aportes metodológicos para el ordenamiento territorial de Montería
}

Quality of urban life and the physical spatial dimension of public space: methodological contributions to the management and urban planning

\author{
Rosana Garnica Berrocal ${ }^{1}$ \\ Juan Eduardo Jiménez Caldera ${ }^{2}$
}

\section{Resumen}

Este artículo analiza las condiciones físico-espaciales del espacio público, que permiten la integración y el encuentro ciudadano, según lo reglamentado en el Decreto 1504 de 1998, el cual concibe al espacio público como un indicador de calidad de vida urbana, que refleja la satisfacción de necesidades materiales e inmateriales de los individuos a partir del uso y disfrute de sus elementos. Se considera la evaluación objetiva de su infraestructura urbana a partir de la clasificación del mismo, la medición del déficit cuantitativo y la determinación de su distribución en función de la accesibilidad peatonal como soporte fundamental en el ordenamiento territorial, al incorporar el análisis espacial como herramienta para la comprensión de los fenómenos espaciales. La calidad de vida de los habitantes de la ciudad de Montería es afectada por las condiciones físico-espaciales del espacio público que permiten el desarrollo de actividades para el esparcimiento, la recreación y el encuentro ciudadano.

1 Magíster en Geografía, Universidad de Córdoba. Docente ocasional de tiempo completo, Universidad de Córdoba. Colombia. garna79@yahoo.es

2 Geógrafo, Universidad de Córdoba. Docente de Cátedra, Universidad de Córdoba. Colombia. juan_eduardo85@yahoo.es 
Palabras clave: calidad de la vida, necesidades básicas, planificación, planificación urbana, espacio público.

\section{Abstract}

This article discusses the physical and spatial conditions of public space that allow integration and citizen gatherings, as regulated in Decree 1504 of 1998, which conceives of public space as an indicator of quality of urban life, which reflects the satisfaction of tangible needs and intangible assets of individuals from the use and enjoyment of their elements, considering the objective assessment of urban infrastructure from its classification, quantitative deficit measurement and determination of its distribution considering the pedestrian accessibility as a fundamental support in urban planning to incorporate spatial analysis as a tool for understanding spatial phenomena. The results concluded that in the city of Monteria, the quality of people's life is affected by the absence of physical and spatial conditions of public space to enable the development of activities for passive recreation, active recreation and citizen gatherings.

Keywords: quality of life, basic needs, planning, urban planning, public space. 


\section{Introducción}

La ciudad se convierte en un espacio privilegiado de encuentro físico, relación personal y afectiva, intercambio económico, cultural, social, político e intelectual, tras el fenómeno de urbanización que viene asociado a una ampliación e intensificación de una pobreza urbana absoluta ya crítica en muchos países, entre ellos Colombia. Lo anterior se evidencia en el crecimiento del déficit cuantitativo y cualitativo de vivienda, los asentamientos de origen informal ubicados con frecuencia en zonas de riesgo no mitigable, la periurbanización, la suburbanización y la segregación socioespacial, como también en la insuficiencia de la infraestructura pública, tanto de servicios públicos domiciliarios como de vías, de espacio público físico y equipamiento público.

Ante esta situación, la vida en los espacios urbanos está sometida a distintos agentes productores de estrés (los medios de transporte terrestre, el ruido, la contaminación visual y del aire, entre otros). El incremento del parque automotor, la construcción de vías para dar paso a los vehículos motorizados, el aumento de edificaciones, se convierten en causas principales de estos agentes que transforman continuamente el entorno (UNFPA, 2007). Esto ha justificado por mucho tiempo la existencia de espacios articuladores, de encuentro e intercambio ciudadano en las ciudades, como parques, canchas deportivas, zonas verdes, entre otros sitios para el esparcimiento, la recreación pasiva y activa, y la circulación. Todos destinados al uso de la comunidad en general de forma gratuita y de libre acceso (León \& Naranjo, 20053), los cuales ayudan a los citadinos a contrarrestar las afectaciones que ocasiona el diario vivir en las ciudades y a destinar de manera provechosa y segura el tiempo de ocio.

En tal sentido, el espacio público se considera un elemento de importancia para la valoración de la calidad de vida en la ciudad, debido a que contribuye a la satisfacción de necesidades materiales e inmateriales de los individuos. A su vez, este es un atributo urbano, junto con el suelo, la vivienda, los servicios públicos o los equipamientos. De la misma forma, suele concebirse como un equipamiento público físico concreto, destinado a usos específicos, ubicados acá o allá en la trama de la ciudad (Alcaldía Mayor de Bogotá y Programa de las Naciones Unidas, 2007).

En efecto, la calidad de vida urbana se manifiesta de forma favorable en las ciudades, entre otros aspectos, en la medida que existan mínimas condiciones físicas y espaciales que garanticen el uso y disfrute del espacio público, es decir, el buen estado de los componentes que lo conforman, la suficiencia y distribución equitativa de sus elementos en el territorio, la forma como deben estar constituidos (escenarios deportivos, de estancia e infantiles), teniendo en cuenta que estos son utilizados por dife-

3 Ver León y Naranjo (2005), especialmente el ítem ¿es el espacio público cada vez más privado?, para establecer las diferencias entre espacio público y privado. 
rentes usuarios (niños, jóvenes, adultos, ancianos) los cuales, en muchos casos, no comparten los mismos intereses al momento de satisfacer sus necesidades.

En este marco, es válido señalar que la normativa colombiana existente de espacio público carece de una metodología clara que contribuya con la planificación urbana y el ordenamiento territorial, y presenta limitaciones en la medición de indicadores, pues deja de lado el análisis espacial que sirve de instrumento para comprender los fenómenos espaciales.

El primer marco de referencia de la aplicación de lo propuesto en el ámbito metodológico en esta investigación, se evidencia en el Plan de Ordenamiento Territorial de Montería, aprobado mediante Decreto 029 del año 2010, en el cual se acogen los resultados obtenidos. Montería es una ciudad de la región Caribe con población superior a 350.000 habitantes, que al igual que otras ciudades latinoamericanas, gran parte de su territorio se ha originado de manera informal, toda vez que alrededor del $70 \%$ de sus barrios se ha consolidado bajo la ilegalidad, con carencias de espacio público, infraestructuras, equipamientos, servicios públicos, entre otros aspectos, haciendo evidente un déficit cuantitativo de espacio público de $13,35 \mathrm{~m}^{2}$ por habitante, en promedio, si se compara con el estándar urbanístico exigido por la Organización Mundial de la Salud (OMS) de los quince metros cuadrados por habitante. Así, en el área urbana de Montería existen 88 escenarios deportivos (distribuidos principalmente en las comunas cuatro, tres y ocho), 77 es- cenarios infantiles y 125 escenarios de estancia, de los cuales solo uno tiene una cobertura regional (Parque Lineal Ronda del Sinú) y el resto cobertura zonal y barrial. Asimismo, las áreas periféricas de la ciudad presentan la menor accesibilidad al espacio público, principalmente en las comunas cuatro, seis y tres, coincidiendo con las áreas de mayor concentración de la población.

La inequidad en la distribución de los elementos del espacio público en la ciudad de Montería, sugiere una intervención por parte de los entes planificadores. Para ello es fundamental tener presente la composición que deberían tener los elementos del espacio público, así como la ubicación o el lugar estratégico que deben ocupar en el espacio urbano (ciudad, comuna o barrio). De esta manera, los mapas de accesibilidad peatonal constituyen una buena herramienta para el ejercicio de la planificación y el ordenamiento territorial.

De acuerdo con lo anterior, se plantea como discusión la incidencia que tienen o no las condiciones físico-espaciales del espacio público en la calidad de vida de urbana y la importancia que tiene abordar estos aspectos en los procesos de ordenamiento territorial, para lo cual se plantean los siguientes interrogantes: ¿Cuáles son las variables físico-espaciales que se deben considerar en un estudio del espacio público para evaluar la calidad de vida urbana? y ¿Cómo las condiciones físico- espaciales del espacio público permiten el uso y goce de sus elementos constitutivos necesarios 
para la interacción entre el individuo, el ambiente y los demás?

La respuesta a estos interrogantes se logra, en parte, gracias a la revisión bibliográfica de estudios realizados desde diferentes perspectivas (social, sicológica, económica y urbanística) sobre el espacio público, que se centran en ciudades y ámbitos espaciales distintos, como sucede con Barcelona: Ciudad e inmigración: usos y apropiación del espacio público, bajo la autoría de Díaz y Ortiz (2003); Madrid: Accesibilidad a zonas verdes urbanas mediante sistemas de información geográfica (SIG), cuyos autores son Herrero, Pérez y Martín (2001); en el ámbito local con Montería imaginada: una ciudad vivi$d a$, de Restrepo (2007); asimismo, con Colombia a partir de La dimensión social del espacio público. Aportes para la calidad de vida urbana, de Páramo y García (2010). También han sido objeto de diseño de planes como instrumentos de planificación del espacio público, las ciudades de Sevilla, Bogotá, Medellín, entre otras, notándose la ausencia del análisis espacial que integre variables físico-espaciales que demuestren las condiciones del espacio público y su incidencia en la calidad de vida urbana.

En esta investigación se identifican y clasifican los elementos del espacio público según su composición, considerando que estas composiciones deben estar distribuidas en todo el territorio para que los diversos usuarios, gocen o disfruten de ellos sin ser excluidos. Igualmente, se determina el déficit cuantitativo que mide la carencia o insuficiencia en la disponibilidad de elementos de espacio público con relación al número de habitantes permanentes del territorio, el cual muestra si hay o no presión y usos intensivos hacia los elementos existentes, lo cual puede afectar el desarrollo de las actividades que en estos se desarrollan.

Finalmente, se establece el grado de distribución del espacio público en función de la accesibilidad peatonal, para ver cómo están dispuestos sus elementos en el territorio, sea de forma equitativa, equilibrada o viceversa, teniendo en cuenta lo señalado por Scott-Webber y Marshall-Baker (1998, p. 10) sobre la existencia de equidad "cuando los ciudadanos tienen igual oportunidad de interactuar o afectar sus ambientes". Además, de que la calidad de vida con relación a este componente urbano, se manifiesta de forma positiva siempre y cuando se conciban a estos espacios como sitios que permitan el acceso a los diferentes grupos sociales como niños, jóvenes, adultos y ancianos (Jiménez, 2009).

\section{Fundamentos conceptuales $y$ metodológicos}

En este artículo se aborda la ciudad como un fenómeno espacial en el cual se manifiestan la expresión cultural y la construcción social. Hace referencia a la dimensión física del espacio público, sobre su importancia para la calidad de vida y la convivencia ciudadana (Pá- 
ramo \& García, 20104). La ciudad se entiende como un producto físico, una concentración de poblaciones y de actividades, una mezcla social y funcional con capacidad de autogobierno y ámbito de participación simbólica y de participación cívica (Giraldo, 2003). La ciudad, bajo esta visión, se concibe como un lugar de encuentro, de intercambio no meramente económico sino también cultural, ciudad de lugares y de expresión de los colectivos sociales. Es aquí donde el espacio público se asume como espacio para lo "público" que propicia el encuentro, la comunicación y la participación entre los ciudadanos. Yory (2007) (citado por García, 2010, p. 52) piensa que es necesario "indagar acerca del papel del espacio público en la formación de una conciencia respecto de "lo público", entendido como construcción colectiva de orden y satisfacción en la convivencia social donde convergen intereses del bien común". En este sentido, la convivencia ciudadana posibilita el encuentro, la comunicación, el uso y disfrute del espacio público sin importar las diferencias culturales, los orígenes, el nivel social y educativo en espacios favorables para ello (García, 2010).

Lo anterior evidencia una perspectiva interesante pero diferente al espacio público-público visto desde la complejidad del lugar, de la ontología de la complejidad, entendiendo el espacio público como el espacio de las significa-

4 Sobre la importancia de considerar el espacio público como una de las dimensiones de la espacialidad o del territorio para tener en cuenta en los estudios de calidad de vida urbana, vea Páramo (2010, p. 15-26); para complementar, vea también Páramo y García (2010). ciones imaginarias, las cuales pueden o no tener como medio para su acceso un espacio público físico que no hay duda del papel que juega en la distribución y apropiación de los bienes públicos y en una mayor integración social, sin ser garantía de una vida política democrática.

Cabe anotar que esta investigación es la primera aproximación al espacio público, por tanto debería ser complementada posteriormente en otras investigaciones mediante la concepción de la dimensión social, de espacio público económicosocial, estético-ambiental y político, de tal forma que se haga énfasis en las interacciones y lógicas no físicas específicas de las distintas dimensiones tanto de la sociedad como de la ciudad.

En el campo de la ciencia geográfica, la ciudad ha sido siempre objeto de la geografía urbana, la cual tuvo su auge desde el momento en que los geógrafos comprendieron que el mundo cada vez tendía a ser más urbano. Desde el punto de vista de la geografía, el tratamiento del espacio público como objeto de estudio en las investigaciones urbanas, es reciente, a pesar de que este componente urbano ha estado en la ciudad desde sus orígenes cuando la plaza, lugar de integración e intercambio ciudadano por excelencia, era considerado el centro de la ciudad, y partir de ella se organizaba la misma. Para comprender de qué forma y en qué momento el espacio público articulador, de intercambio y encuentro ciudadano llegó a ser el eje de los estudios geográficos urbanos, es convenien- 
te hacer seguimiento cronológico sobre el estudio de las ciudades y la incorporación del espacio público en el análisis urbano, lo cual no es el objeto central de esta investigación.

En la actualidad, es tal la importancia que se le atribuye al espacio público, que algunos analistas de las ciudades lo tratan para determinar, si existe o no, crisis internas en ellas (Borja, 19985). El espacio público se convierte en un paradigma emergente que requiere de una gama de discusiones, entre otras, enmarcadas en las nuevas leyes de desarrollo urbano que arrojan una noción de espacio público desde la nueva significación cultural y urbana.

En este orden de ideas, se plantean discusiones sobre lo público, la forma como nos hacemos humanos a través del lugar y cómo el lugar coadyuva a ese proceso de hacernos humanos (Giraldo, 2003). Hoy en día, la geografía urbana ha conservado parte de sus tradicionales formas de ver y comprender los espacios urbanos. Sin embargo, la dinámica interna de las ciudades actuales ha orientado los estudios hacia la comprensión de los espacios y sistemas que la conforman, (edificaciones, calles, barrios, parques, etc.), analizando los desarrollos desiguales o desequilibrios que perjudican a la sociedad que la integra.

Los elementos de análisis relacionados con las condiciones físicas y espaciales

5 Borja (1998) reitera entre los avatares de la modernidad, que se ha olvidado hacer ciudad como producto integral e integrador y con ello, el espacio público. 0 por lo menos se relega a un rol secundario. que deben existir para que el espacio público articulador, de intercambio y encuentro ciudadano contribuya a mejorar la calidad de la población citadina, se fundamentan en el modelamiento espacial de distintas variables ( clasificación de los elementos constitutivos del espacio público según su composición y escala; el déficit en términos de suficiencia, es decir, superficie o área y la distribución espacial basada en la accesibilidad peatonal), algunas de las cuales se miden mediante indicadores y otras a través de representaciones espaciales sobre el territorio urbano objeto de estudio, las cuales han sido consideradas en la presente investigación para aproximarse al estudio de la dimensión física del espacio público y su incidencia en la calidad de vida urbana, sirviendo de insumo base de diagnóstico para la toma de decisiones en los procesos de ordenación territorial.

\subsection{Fases de la investigación}

La investigación se desarrolla en tres fases, como se describe a continuación:

\subsubsection{Clasificación de los elementos del espacio público articulador, de inter- cambio y encuentro ciudadano}

En esta se reconoce la constitución o conformación física (a modo de diagnóstico) de cada uno de los elementos del espacio público presentes en un territorio como factor clave para determinar en unidad de superficie a los espacios que están siendo aprovechados debida- 
mente por los diferentes tipos de usuarios (niños, jóvenes, adultos y adultos mayores), de tal manera que se logren satisfacer las necesidades básicas asociadas al tiempo de ocio y recreación en el marco de un proceso de planificación urbana y el ordenamiento territorial. La clasificación del espacio público según su composición y escala, se realizó con base en siete criterios (Tabla 1).

Tabla 1. Criterios de clasificación del espacio público de articulación y encuentro ciudadano según su composición en Montería

\begin{tabular}{|c|c|c|}
\hline $\begin{array}{l}\text { CLASIFICACIÓN DEL } \\
\text { ESPACIO PÚBLICO SEGÚN } \\
\text { SU COMPOSICIÓN }\end{array}$ & FUNCIÓN & DESCRIPCIÓN \\
\hline Escenarios deportivos & \multirow[b]{2}{*}{ Ocio y recreación activa } & $\begin{array}{l}\text { Superficie destinada únicamente al ejercicio de la actividad } \\
\text { deportiva de la comunidad en general. (Canchas de fútbol, } \\
\text { baseball, basketball, tejo, etc.). }\end{array}$ \\
\hline Escenarios infantiles & & $\begin{array}{l}\text { Superfici e que, p or s us e lementos c onstituyentes } \\
\text { (equipamient o infantil t ales c om o columpios, } \\
\text { subibajas., etc.), d estinada únicamente a } 1 \text { ejercicio de la } \\
\text { actividad recreativa para niños. }\end{array}$ \\
\hline Escenarios de estancia & $\begin{array}{l}\text { Ocio, recreación pasiva, reunión y } \\
\text { armonía }\end{array}$ & $\begin{array}{l}\text { Superficies constituidas p or aspectos ambientales como } \\
\text { árboles (suministro des ombra), césped y, dotado con } \\
\text { mobiliario como bancas; destinado para el ejercicio de la } \\
\text { actividad pasiva (descanso y la armonía de la población en } \\
\text { general.) }\end{array}$ \\
\hline \multicolumn{3}{|c|}{$\begin{array}{l}\text { Es posible encontrar elementos d } 1 \text { espacio público con presencia de dos o tres de los escenarios, lo que se conoce como espacio } \\
\text { úblico diverso. Entre más com osiciones ten a un elemento tres co o máximo se considera más diverso. }\end{array}$} \\
\hline $\begin{array}{l}\text { Espacio público compuesto por } \\
\text { escenarios deportivos y p arques } \\
\text { infantiles }\end{array}$ & \multirow{4}{*}{$\begin{array}{l}\text { Ocio, recreación activa y pasiva, } \\
\text { reunión y armonía }\end{array}$} & $\begin{array}{l}\text { Superficie multifuncional, destinada a la recreación infantil } \\
\text { y a la práctica de la actividad deportiva. }\end{array}$ \\
\hline $\begin{array}{l}\text { Espacio público compuesto por } \\
\text { espacios verdes d e estancia y } \\
\text { parques infantiles }\end{array}$ & & $\begin{array}{l}\text { Superficie multifuncional, destinada a la recreación } \\
\text { infantil y al descanso y la armonía de } 1 \text { a población en } \\
\text { general. }\end{array}$ \\
\hline $\begin{array}{l}\text { Espacio público compuesto por } \\
\text { espacios verdes } \mathrm{d} \text { e estancia y } \\
\text { escenarios deportivos }\end{array}$ & & $\begin{array}{l}\text { Superficie multifuncional, destinada a al descanso y la } \\
\text { armonía de la población en general y a la práctica de la } \\
\text { actividad deportiva }\end{array}$ \\
\hline $\begin{array}{l}\text { Espacio público compuesto por } \\
\text { espacios v erdes de e stancia, } \\
\text { parques infantiles y escenarios } \\
\text { deportivos }\end{array}$ & & $\begin{array}{l}\text { Superficie multifuncional, destinada a la recreación } \\
\text { infantil, al d escanso y la armonía de } 1 \text { a población en } \\
\text { general y a la práctica de actividades deportivas. }\end{array}$ \\
\hline
\end{tabular}

Fuente: elaboración propia con base en trabajo de campo.

Tabla 2. Criterios de clasificación del espacio público de articulación y encuentro ciudadano, existentes según la escala

\begin{tabular}{|l|l|}
\hline CLASIFICACIÓN SEGUN ESCALA & \multicolumn{1}{|c|}{ DESCRIPCIÓN } \\
\hline Espacio público de escala regional & $\begin{array}{l}\text { Son espacios de gran dimensión y altos valores } \\
\text { ambientales, que satisfacen necesidades a } \\
\text { población local y de otras localidades distantes. }\end{array}$ \\
\hline Espacio público de escala zonal & $\begin{array}{l}\text { Areas o espacios libres, con una dimensión } \\
\text { variable, destinada a la satisfacción de necesidades } \\
\text { de recreación activa y/o pasiva de un grupo de } \\
\text { barrios. }\end{array}$ \\
\hline Espacio público de escala vecinal & $\begin{array}{l}\text { Areas o espacios libres, destinados a la recreación, } \\
\text { 1a reunión y la integración de la comunidad, que } \\
\text { cubren necesidades de barrios. Se les domina } \\
\text { genéricamente parques, zonas verdes o cesiones } \\
\text { para parques. El parque de bolsillo es una } \\
\text { modalidad de parque vecinal, que tiene un área } \\
\text { inferior a 1.ooo me destinado exclusivamente a la } \\
\text { recreación pasiva contemplativa. }\end{array}$ \\
\hline
\end{tabular}

Fuente: elaboración propia a partir de la clasificación realizada por la Alcaldía Mayor de Bogotá en el POT vigente, en su artículo 230 (Título II Componente urbano, subtítulo 3, capítulo 10 Sistema de espacio público construido). 
En síntesis, dos elementos de análisis se originan a partir de la clasificación de los elementos del espacio público. El primero es el porcentaje de presencia del espacio público (porcentaje PPEP), indicador que otorga una idea global sobre como es la distribución de los diversos elementos del espacio público presentes la ciudad. La fórmula de PPEP es la siguiente:

$$
\text { PPEP }=\frac{\text { \# Elementos del EP según su composición }}{\text { \# Total elementos EP }} \times 100
$$

El segundo elemento de análisis es netamente descriptivo, referido a la distribución de los elementos del espacio público según la escala y el impacto de su ubicación en el territorio urbano.

\subsubsection{Determinación del déficit cuanti- tativo de espacio público}

Cabe anotar que la carencia de espacio público es un factor que conlleva a la presión o sobreutilización de sus elementos por quienes acuden a recrearse pasiva o activamente, o a destinar el tiempo de ocio; siendo esto un obstáculo para el desarrollo óptimo de las actividades que se van a ejercer. El déficit cuantitativo de espacio público (DCEP) indica "la carencia o insuficiente disponibilidad de elementos de espacio público con relación al número de habitantes permanentes del territorio”, según el Decreto 1504 de 1998 en su artículo 12. Se obtiene al contrastar el indicador resultante de la fórmula "AHEP = EP/\#hab. y el índice óptimo estándar (IOSEP) establecido por entes internacionales y locales de $15 \mathrm{~m}^{2} /$ habitante. El valor que resulta de la resta entre estos dos valores, es el déficit cuantitativo, en otros términos, DCEP $=($ AHEP - IOSEP $)$. Comúnmente el DCEP es expresado en metros cuadra- dos de espacio público por habitante $\left(\mathrm{m}^{2} /\right.$ hab. $)$.

En consecuencia con lo anterior, el espacio público debe ser considerado un indicador de calidad de vida urbana, que a partir de la evaluación objetiva de sus infraestructuras (escenarios deportivos, recreativos y de estancia), amoblamiento urbano (bancas, luminarias, baños públicos, entre otros) y la accesibilidad peatonal, trascienda la evaluación subjetiva con la consideración de la percepción de los ciudadanos sobre la función que cumplen esos mismos elementos para el deporte, la recreación, la integración social, la relación con los demás, las celebraciones culturales, las manifestaciones sociales, la integración entre los distintos componentes del espacio público, la justicia social que favorece la accesibilidad, la calidad de los servicios y la estética del paisaje, entre otros aspectos (Páramo, 2007).

\subsubsection{Determinación del grado de dis- tribución de los elementos del espacio público}

Para llevar a cabo esta fase, se requiere entender que el grado de distribución de los elementos del espacio público (distribución equitativa o equilibrada y vi- 
ceversa) depende de la accesibilidad peatonal hacia los mismos, es decir, de las rutas de acceso existentes y de qué tanta sea la distancia que deben recorrer los individuos para acceder a éstos.

En el ámbito general se sugiere el diseño de espacios verdes y otros elementos del espacio público que permitan a los ciudadanos vivir a una distancia no mayor de quince minutos caminando, es decir, un poco más de $600 \mathrm{~m}$ y menos de $1000 \mathrm{~m}$, considerando que una persona camina $4 \mathrm{~km} /$ hora, aproximadamente (Flores \& González, 2007). En el estudio de accesibilidad a zonas verdes urbanas realizado en Madrid, España (2001), se cree que hay buena accesibilidad en aquellas áreas que se encuentran dentro de un radio de influencia de $300 \mathrm{~m}$ alrededor de las zonas verdes (no más de cinco minutos caminando).

En este sentido, la distancia es un factor esencial del cual depende la asistencia de ciertos tipos de usuarios del espacio público, como niños o adultos mayores, que por sus condiciones físicas y vulnerabilidad, se ven desfavorecidos al hacer extensos recorridos para gozar de los beneficios que ofrecen los escenarios infantiles, deportivos y de estancia distribuidos en las ciudades, lo cual muestra la importancia de planificar el entorno físico hacia la satisfacción de las necesidades de ocio, recreación, deporte, socialización, aprendizaje de la cultura, el encuentro con la naturaleza y la convivencia entre extraños. Por tal razón, 300 metros se considera la distancia prudente y adecuada para la construcción de este tipo de espacios.

La Figura 1 ilustra el modelo conceptual de la accesibilidad peatonal al espacio público. En esta se aprecia que el número de rutas de acceso es otro factor que determina la accesibilidad peatonal, además de la distancia, teniendo en cuenta para el caso del primero, que al menos una ruta debe existir como conector para establecer que hacia un elemento del espacio público hay acceso, y para el segundo, se busca determinar el grado de acceso (bueno o malo). Hay que anotar que la distribución del espacio público es equilibrada o equitativa en el territorio, si y solo si, toda la población posee buena accesibilidad peatonal a los diversos elementos que lo conforman; y viceversa, si el total o parte de la población no accede a dichos elementos.

La mayoría de la información y datos empleados en esta investigación, son de tipo primario, producto de la recolección en campo mediante la técnica de la observación, y, en menor medida, de tipo secundario, suministrado por fuentes de información como Parques Nueva Montería (concesión encargada de la administración y mantenimiento de los parques durante el tiempo en que se efectuó el estudio), Proactiva S.A (empresa prestadora del servicio de acueducto y aseo), DANE 2005 (Departamento Administrativo Nacional de Estadística), Alcaldía municipal de Montería (Secretaría de Planeación Municipal), entre otras fuentes. 


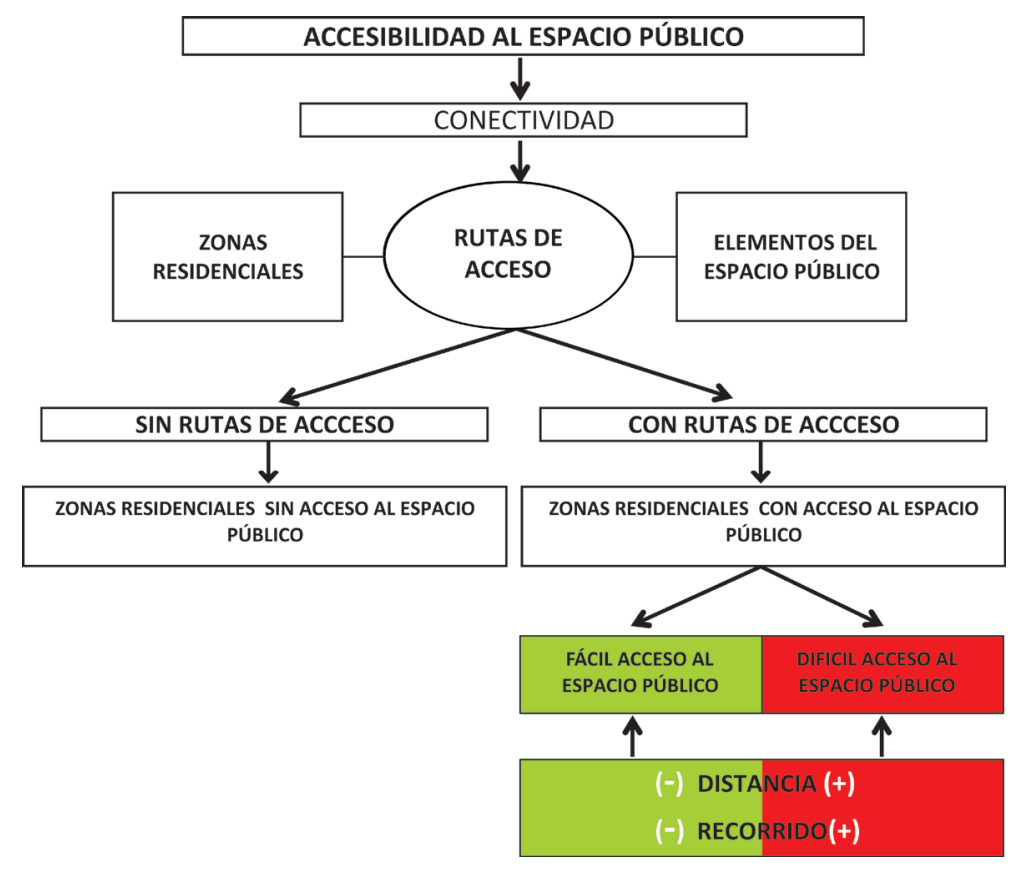

Figura 1. Modelo conceptual para la accesibilidad peatonal al espacio público.

Fuente: elaboración propia a partir del estudio de accesibilidad de zonas verdes en Madrid (2001).

\section{Resultados y discusión}

Montería, en su área urbana, cuenta con 185 elementos del espacio público articulador, de encuentro e intercambio ciudadano, distribuidos en las nueve comunas existentes en ella, (las cuales son las unidades mínimas para el ordenamiento en el nivel local de este importante componente urbano), según determina el Plan de Ordenamiento Territorial de Montería (POT) 2002-2011. Dichos elementos están compuestos por escenarios infantiles, de estancia y deportivos representados de forma individual o combinando dos o tres de ellos en una sola estructura. A su vez, estos mantienen una influencia sobre la población, cubriendo las necesidades en el ámbito vecinal, zonal y regional.

\subsection{Clasificación del espacio público según su composición y escala}

Los elementos del espacio público articulador, de intercambio y de encuentro ciudadano, adquieren denominaciones tales como: parques, canchas deportivas, rondas, zonas verdes, entre otros, las cuales van de acuerdo con sus características físicas. La práctica de actividades que contribuyen al mejoramiento de la calidad de vida como el esparcimiento pasivo, la recreación activa, son vivenciadas por los diversos usuarios 
(niños, jóvenes, adultos, ancianos), quienes mantienen intereses o preferencias individuales, diferentes entre sí, al momento de satisfacer sus necesidades en lo que tiene que ver con el espacio público (Jiménez, 20096).

Para que la calidad de vida urbana, principal objetivo del espacio público articulador, de intercambio y encuentro ciudadano, se manifieste de manera favorable, es conveniente que en las ciudades, la presencia de escenarios deportivos, de estancia e infantiles, en cualesquiera de sus denominaciones (parque, cancha deportiva, ronda, etc.), se distribuya en todo el territorio, lo cual contribuye a que los diversos usuarios disfruten de ellos sin ser excluidos.

Con base en lo anterior, los elementos del espacio público existentes en la ciudad de Montería se han clasificado según la presencia de las composiciones (Tabla 3 y Figura 2). La Tabla 3 registra la presencia de elementos del espacio público compuestos por escenarios de estancia, siendo mayor con relación a los demás escenarios, en seis de las nueve comunas (uno, tres, cinco, seis, siete y ocho), de las cuales se destaca la comuna siete, con un porcentaje de presencia del $100 \%$, indicando que todos los elementos del espacio público tienen áreas destinadas a la práctica de actividades pasivas. Seguidamente

6 En la tesis de pregrado de Geografía, Jiménez (2009) señala la diversidad de los espacios públicos según la clasificación de composición y escala, las cuales tienen sus propias características físicas y permiten la realización de distintas actividades humanas. se registran los valores de las comunas uno y seis donde el número de elementos con esta composición doblega a las otras, con porcentajes de presencia del $70.96 \%$ y $82.76 \%$ respectivamente.

El porcentaje de presencia en los escenarios deportivos es mayor en las tres comunas restantes, (dos, cuatro y nueve), con valores de 56,25 \%, 70.00 $\%$ y $54.55 \%$ respectivamente; sin embargo, estos valores, al resultar bajos, son poco significativos, si se tiene en cuenta el total de elementos del espacio público distribuidos en estas zonas. Esto mismo ocurre con los escenarios infantiles, que son de poca representación al observar el total.

La presencia en mayor porcentaje de escenarios de estancia entre los elementos del espacio público, está presumiendo que la ciudad le apuesta a la generación de espacio para un grupo de habitantes, que solo permite el desarrollo de actividad pasiva, lo que excluye a la población practicante del deporte y actividades relacionadas con la recreación infantil. Esto revela en cierto modo que la planificación urbana orientada a estos espacios, no está siendo justa. Lo ideal es satisfacer a toda la población, mientras exista simetría en las composiciones de los elementos que conforman el espacio público articulador, de intercambio y encuentro ciudadano asumiendo la presencia en la ciudad de una sociedad diversa (haciendo referencia a los grupos etáreos que la conforman). 
Tabla 3. Clasificación del espacio público según su composición

\begin{tabular}{|c|c|c|c|c|c|c|c|}
\hline \multirow{2}{*}{$\begin{array}{c}\text { COMU } \\
\text { NA }\end{array}$} & \multirow{2}{*}{$\begin{array}{c}\text { Elementos del } \\
\text { Espacio Público } \\
\text { Articulador y de } \\
\text { Encuentro } \\
\text { Ciudadano }\end{array}$} & \multicolumn{3}{|c|}{$\begin{array}{l}\text { Presencia de Escenarios en los } \\
\text { Elementos del Espacio Público }\end{array}$} & \multicolumn{3}{|c|}{$\%$ de Presencia } \\
\hline & & $\begin{array}{l}\text { Escenarios } \\
\text { Deportivos } \\
\text { (ED) }\end{array}$ & $\begin{array}{l}\text { Escenarios } \\
\text { Infantiles } \\
\quad \text { (EI) }\end{array}$ & $\begin{array}{l}\text { Escenarios } \\
\text { de Estancia } \\
\text { (EE) }\end{array}$ & $\%$ ED & $\%$ EI & $\%$ EE \\
\hline 1 & 31 & 11 & 11 & 22 & 35,48 & 35,48 & 70,97 \\
\hline 2 & 16 & 9 & 8 & 8 & 56,25 & 50,00 & 50,00 \\
\hline 3 & 21 & 14 & 5 & 15 & 66,67 & 23,81 & 71,43 \\
\hline 4 & 30 & 21 & 17 & 16 & 70,00 & 56,67 & 53,33 \\
\hline 5 & 16 & 7 & 8 & 13 & 43,75 & 50,00 & 81,25 \\
\hline 6 & 29 & 7 & 10 & 24 & 24,14 & 34,48 & 82,76 \\
\hline 7 & 8 & 1 & 4 & 8 & 12,50 & 50,00 & 100,00 \\
\hline 8 & 23 & 12 & 11 & 14 & 52,17 & 47,83 & 60,87 \\
\hline 9 & 11 & 6 & 3 & 5 & 54,55 & 27,27 & 45,45 \\
\hline Total & 185 & 88 & 77 & 125 & 47,57 & 41,62 & 67,56 \\
\hline \multicolumn{8}{|c|}{$\begin{array}{l}\text { Nota 1: Fueron excluidos aquellos elementos del espacio público que están en proceso de remodelación, } \\
\text { así como áreas libres (lotes baldíos) incluidos en planes para creación de nuevos espacios. Nota 2: Los } \\
\text { porcentajes de presencia son estimados con relación al número total de elementos del espacio público por } \\
\text { comunas. }\end{array}$} \\
\hline
\end{tabular}

Fuente: elaboración propia con fundamento en el Decreto 1504 de 1998.

En la mayoría de los casos, los ciudadanos se ven obligados a desplazarse a otras comunas para realizar prácticas deportivas y recreativas debido a la escasez de estos elementos del espacio público. Asimismo, en Montería sólo existe un elemento de espacio público de escala regional como lo es el Parque Lineal Ronda del Sinú, que atrae la atención de los habitantes de la ciudad, de localidades vecinas y de turistas que van de paso por la ciudad, como se observa en la Figura 3.

En este sentido, el Parque Lineal Ronda del Sinú, se convierte en un elemento de integración urbanística y de reconocimiento a nivel nacional y regional, como un lugar de encuentro por excelencia que requiere de acciones integra- les en materia de ordenación del territorio y de la gestión del mejoramiento de la calidad del medio ambiente, de tal modo que se impacte la movilidad y se articulen las edificaciones y la vivienda a través del espacio público en el resto de la ciudad. Es así como el Plan de Ordenamiento Territorial, POT de Montería 2002-2011 en el Acuerdo 018 de 2002 (artículo 20), propuso un imaginario de ciudad "socialmente equilibrada e integrada espacial y funcionalmente", a partir del río Sinú, al ser uno de los elementos del medio natural, entre otros, que determinan la morfología y calidad espacial urbana.

En Montería es común hallar en mayor proporción elementos clasificados en la categoría vecinal, los cuales se originan 


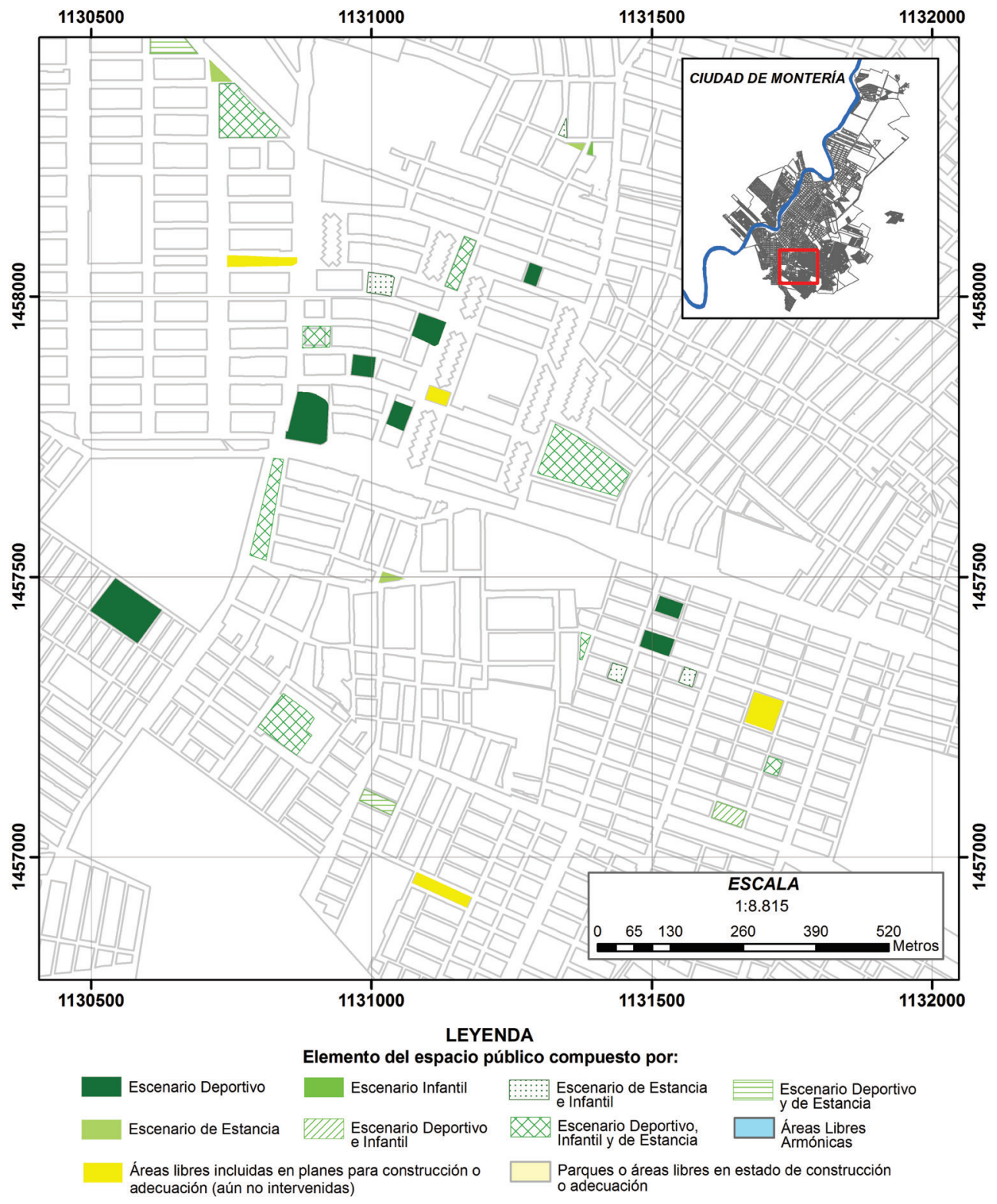

Figura 2. Mapa de clasificación del espacio público según su composición.

Fuente: elaboración propia con base en trabajo de campo y el Decreto 1504 de 1998.

en la construcción de los barrios. A medida que se construyen más de ellos, de forma planificada, la probabilidad de encontrar elementos articuladores y de encuentro ciudadano a escala vecinal, es alta. En el área urbana existen 136 
elementos del espacio público de escala vecinal, esto refleja que la dimensión del espacio público en la ciudad es principalmente barrial, como se ilustra en la parte occidente de la ciudad (Figura 3).
En menor proporción, se encuentra el espacio público de escala zonal (48), concentrado principalmente en las comunas cuatro, tres, cinco y ocho, sobresaliendo los escenarios deportivos,

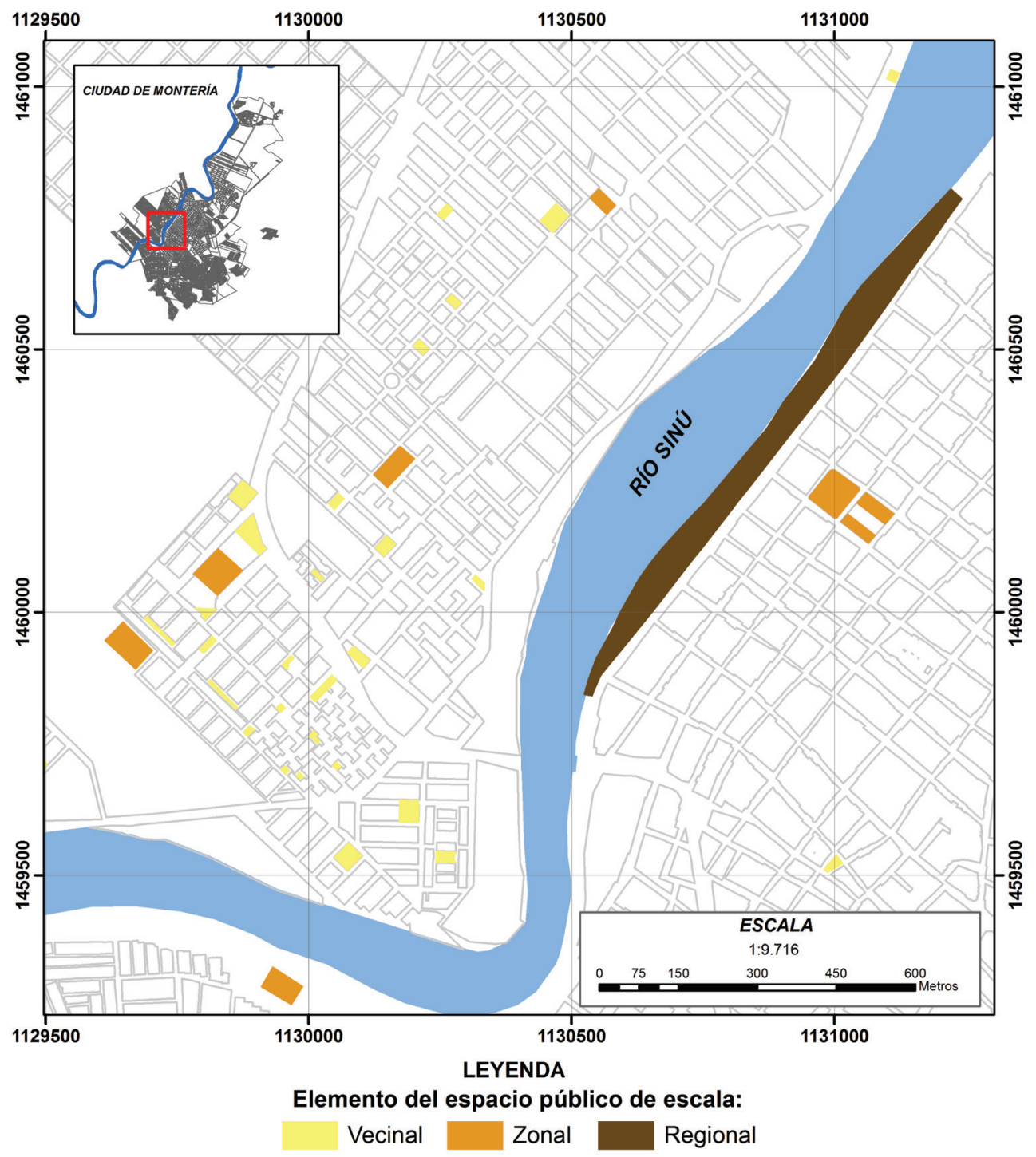

Figura 3. Mapa de clasificación del espacio público según la escala.

Fuente: elaboración propia 
de los cuales se registran en total 32, que corresponden a canchas de fútbol, minifútbol o microfútbol de arena (destacándose en las comunas cuatro, tres y ocho; siete de béisbol o softball, una de tejo y una pista de bicicross).

\subsection{Déficit cuantitativo de espacio pú- blico en el área urbana de Montería}

El déficit cuantitativo de espacio público en el área urbana es de 13,35 m2/habitante, en promedio, indicador que demuestra como la calidad de vida urbana es afectada a causa de la problemática del espacio público en lo que respecta a la insuficiente disponibilidad y carencia de los elementos constitutivos de este para la articulación y el encuentro ciudadano, lo cual dificulta en Montería la satisfacción de las necesidades en la interacción entre el individuo, el ambiente y los otros.

La comuna cinco (centro) registra el menor déficit (11.02 $\mathrm{m} 2 / \mathrm{hab}$.), debido a la presencia del Parque Lineal Ronda del Sinú, un elemento de escala regional de gran dimensión, aunque sin alcanzar el mínimo exigible según la OMS (15 m2/ hab.). Las comunas uno y seis poseen los índices cuantitativos de espacio público más bajos, pues la mayoría de sus elementos tiene una cobertura vecinal y algunos de sus barrios se han originado de manera ilegal con escasez o nula presencia del espacio público, equipamientos, vías y transporte, entre otros aspectos (Negrete, 2001). Sin duda, la presión y usos intensivos hacia los ele- mentos distribuidos en estas localidades se puede estar manifestando de manera excesiva (Pérez, 2004), lo cual desfavorece notablemente a la población que hace uso de estos espacios.

\subsection{Distribución del espacio público basado en la accesibilidad peatonal}

Para que los beneficios que otorga el espacio público se obtengan de forma equitativa, es conveniente que la variedad de usuarios accedan a los diversos elementos representados por escenarios deportivos, de estancia e infantiles. Por tal razón, sus lugares de residencia deben tener rutas de acceso y estar a una distancia prudente hacia dichos elementos, específicamente a cada uno de los escenarios, ya sea separados o unidos en una sola estructura para garantizar su accesibilidad (Figuras 4, 5 y 6).

Con la determinación de la accesibilidad peatonal en función de la distancia a los diversos elementos del espacio público, se puede establecer cuan equitativa es la distribución de tales elementos en el espacio, ya que esta variable muestra si la población tiene o no igual oportunidad de acudir y practicar las actividades relacionadas con ellos.

La buena accesibilidad no garantiza la suficiencia de elementos de espacio público, ya que ésta depende directamente del déficit cuantitativo. Particularmente, los elementos de espacio público en el área urbana de Montería se encuentran distribuidos inequitativamente, puesto 


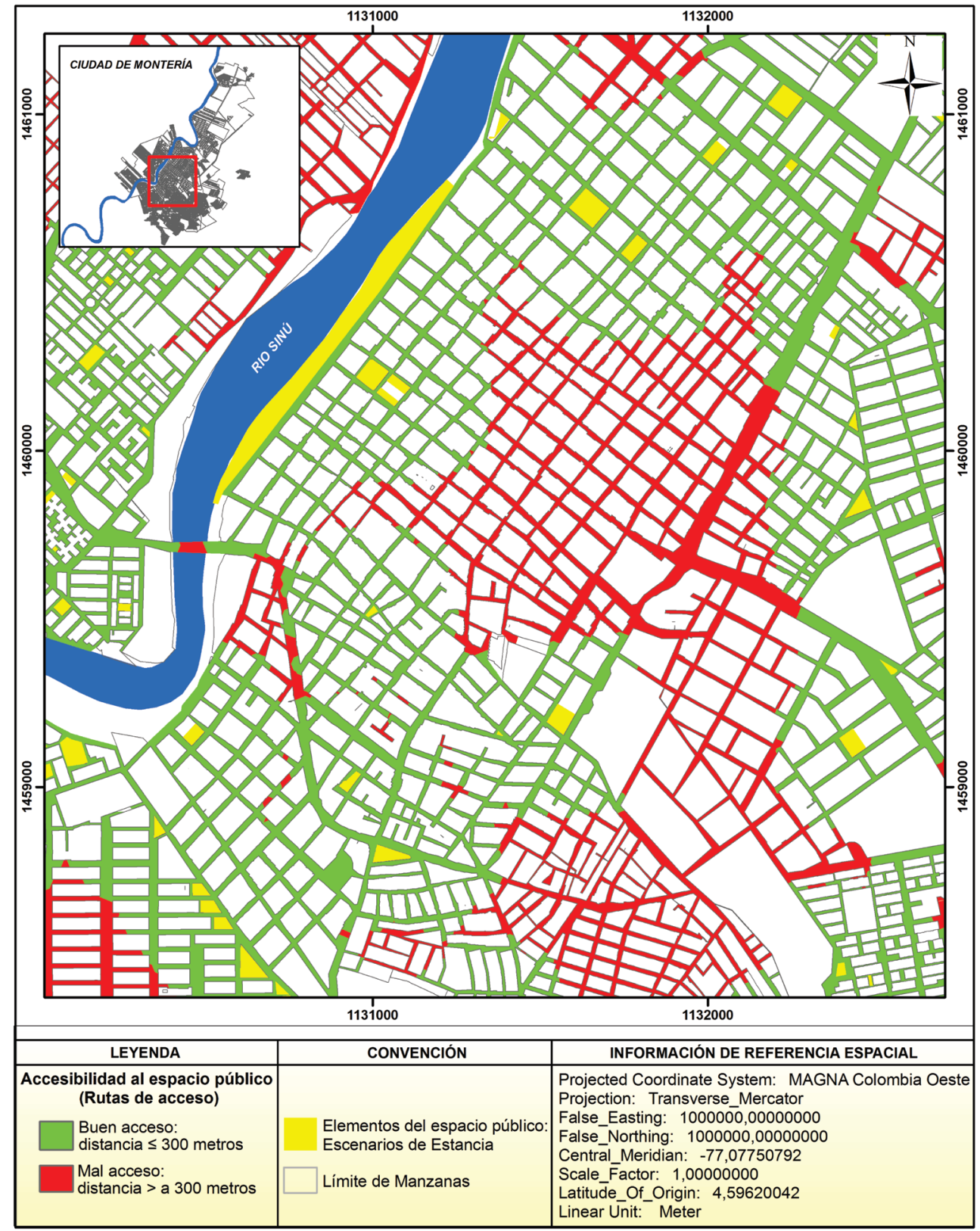

Figura 4. Mapa de accesibilidad peatonal a escenarios de estancia.

Fuente: elaboración propia con base en trabajo de campo. 


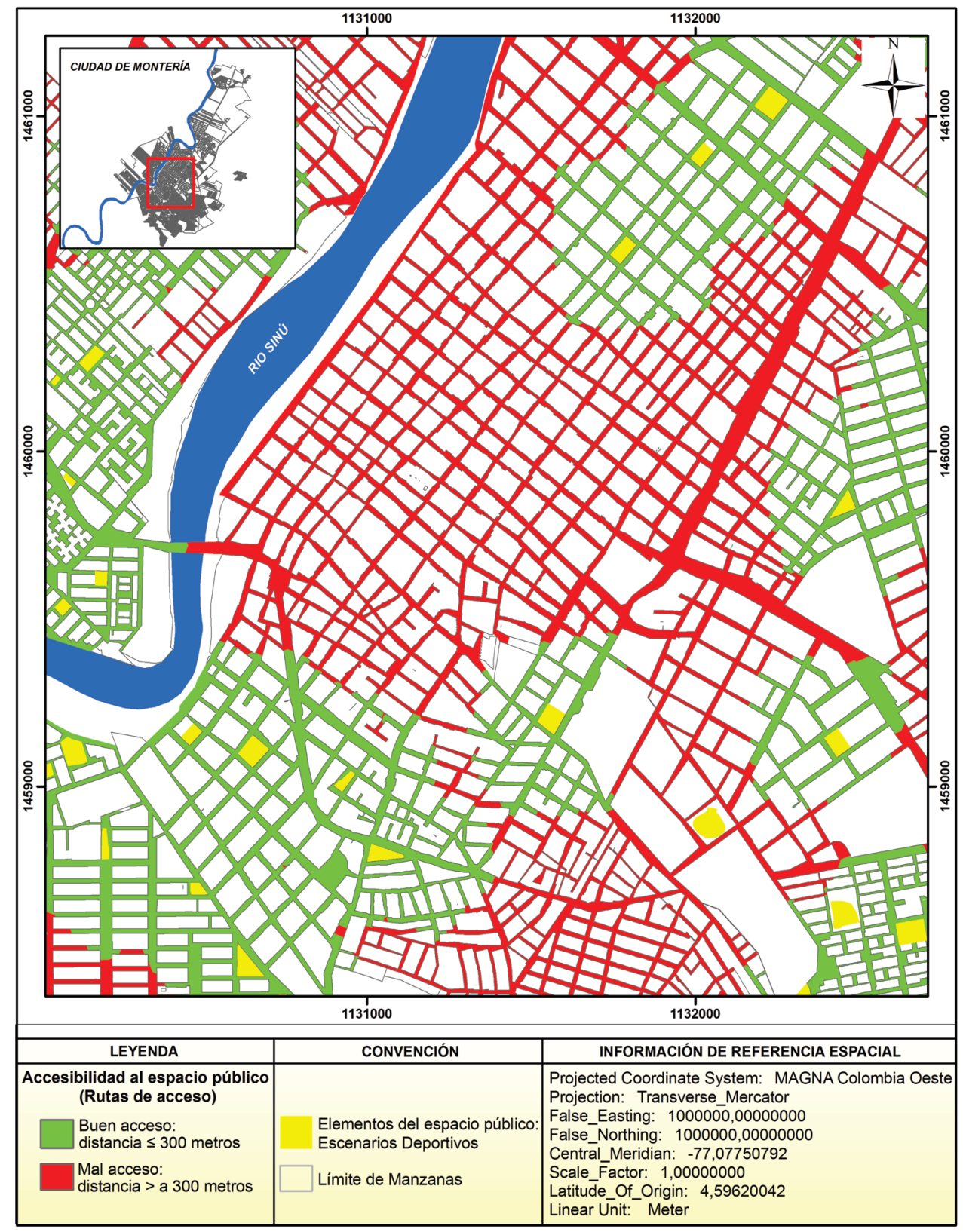

Figura 5. Mapa de accesibilidad peatonal a escenarios deportivos

Fuente: elaboración propia con base en trabajo de campo. 


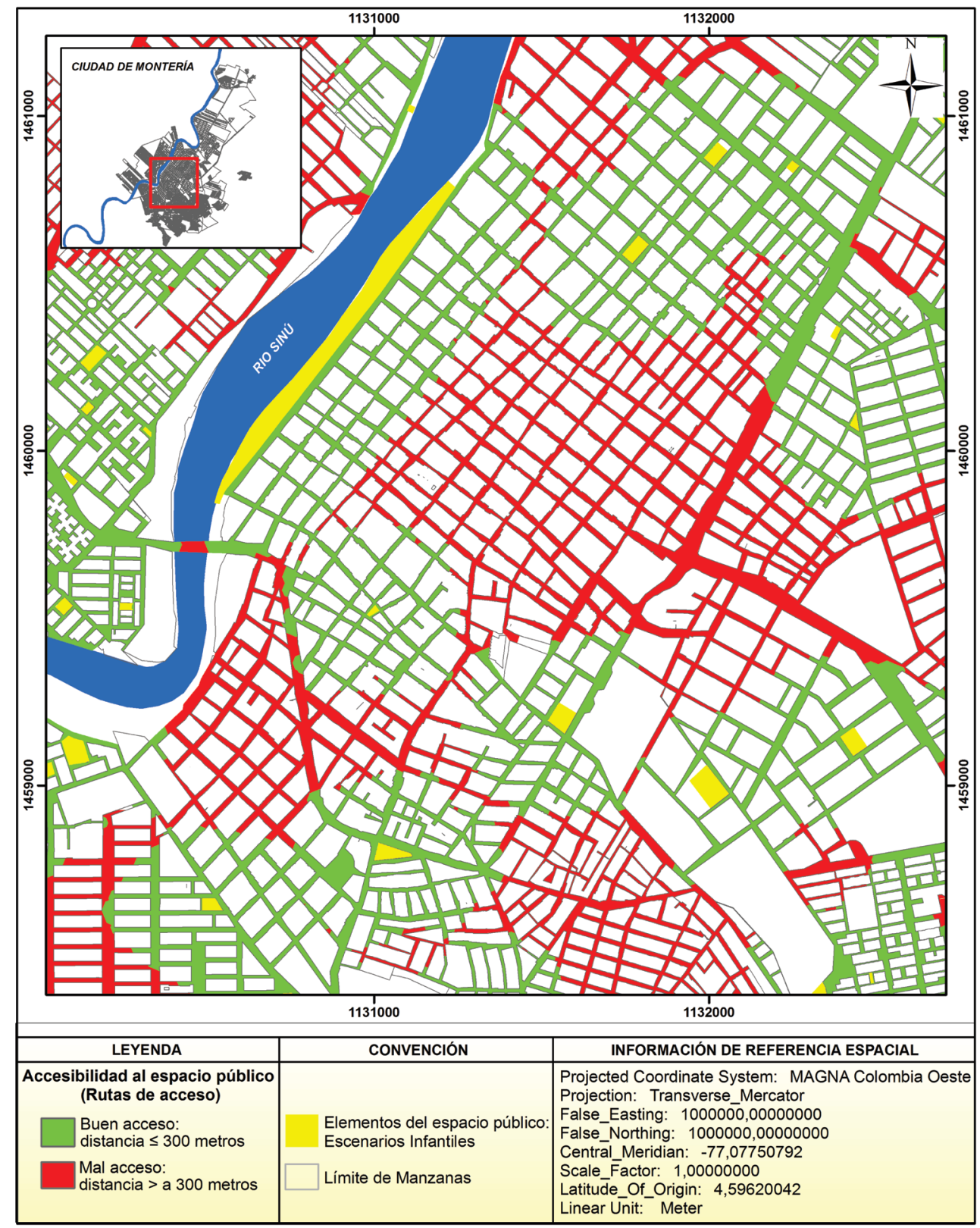

Figura 6. Mapa de accesibilidad peatonal a escenarios infantiles.

Fuente: elaboración propia con base en trabajo de campo. 
que la totalidad de la población no logra acceder adecuadamente al espacio público; en efecto, solo accede óptimamente a espacios compuestos por los tres escenarios, la población residente en 50 barrios de los 152 existentes.

En contraste con lo anterior, los residentes de 28 barrios tienen acceso peatonal a dos espacios públicos diversos. En los barrios restantes (44), la población no accede a ninguno de los tres escenarios (deportivos, de estancia e infantil). Esta situación revela desigualdad social en la ciudad, lo cual afecta la calidad de vida de los ciudadanos, ya que en ciertas zonas los usuarios no obtienen los beneficios que otorgan los espacios articuladores, de intercambio y encuentro ciudadano, por tener muy distantes los elementos con composiciones que complacen sus gustos o intereses, haciéndose notar la insatisfacción de las necesidades tanto materiales como inmateriales de la población (Tabla 4).

Tabla 4. Accesibilidad a los elementos del espacio público según su composición

\begin{tabular}{|c|c|c|c|c|c|c|c|c|}
\hline \multirow{2}{*}{$\begin{array}{c}\text { Nombre } \\
\text { Comuna }\end{array}$} & \multicolumn{6}{|c|}{ \# de Barrios con Accesibilidad a Elementos del Espacio Público Compuesto por } & $\begin{array}{c}\text { \# de Barrios sin } \\
\text { Escenarios: }\end{array}$ \\
\cline { 2 - 9 } & Dep & Est & Inf & Dep + Est & Dep + Inf & Est + Inf & Est + Dep + Inf & Espacio Público \\
\hline 1 & 1 & 0 & 0 & 2 & 0 & 0 & 8 & 3 \\
\hline 2 & 4 & 0 & 4 & 1 & 1 & 1 & 8 & 0 \\
\hline 3 & 0 & 0 & 1 & 0 & 0 & 0 & 4 & 7 \\
\hline 4 & 3 & 0 & 3 & 0 & 5 & 1 & 11 & 12 \\
\hline 5 & 2 & 0 & 0 & 0 & 0 & 7 & 3 & 7 \\
\hline 6 & 0 & 1 & 0 & 0 & 1 & 0 & 3 & 4 \\
\hline 7 & 0 & 0 & 0 & 0 & 0 & 0 & 3 & 2 \\
\hline 8 & 0 & 6 & 1 & 1 & 0 & 6 & 4 & 3 \\
\hline 9 & 0 & 2 & 0 & 2 & 0 & 0 & $\mathbf{5}$ & $\mathbf{4 4}$ \\
\hline
\end{tabular}

Fuente: elaboración propia con base en trabajo de campo.

La Tabla 4 muestra que las comunas tres, cuatro, cinco y seis poseen la mayoría de barrios sin accesibilidad peatonal al espacio público, siendo los de mayor concentración poblacional de la ciudad. En este sentido, la ordenación del territorio debe orientarse de manera prioritaria hacia estas zonas, ya que aquí se afecta en mayor proporción la calidad de vida de los habitantes.

La inequidad en la distribución de los elementos del espacio público en la ciu- dad de Montería, exige una intervención equitativa por parte de los planificadores de este importante componente urbano. Para ello es fundamental tener presente la composición que los elementos del espacio público deben contener, así como la ubicación o el lugar estratégico que deben ocupar en el espacio, sea este la ciudad, comuna o barrio. 


\section{Conclusiones}

La interpretación y análisis de los resultados permiten concluir que en la ciudad de Montería, la calidad de vida de los habitantes se ve afectada por la inexistencia de condiciones físico-espaciales del espacio público que permitan el desarrollo de actividades para el esparcimiento pasivo y la recreación activa. Según Leva (citado por Páramo \& García, 2010), el espacio público se constituye en uno de los satisfactores de la calidad de vida urbana, pues busca cumplir con los requerimientos básicos de sustentabilidad de la vida humana individual y en interacción social dentro del medio urbano.

Con relación a las condiciones físicas del espacio público en Montería, la existencia en mayor proporción de elementos del espacio público compuestos por escenarios de estancia, estaría favoreciendo a la población interesada en realizar actividades tales como: conversar en grupos o parejas, leer o descansar en bancas, entre otras. Esta situación se presenta con más determinación en las comunas uno, cinco, seis y siete, donde los porcentajes de presencia son del $70.97 \%, 81.25 \%, 82.76 \%$ y $100 \%$ respectivamente. En forma contraria, en estas mismas localidades existen pocos espacios destinados a la recreación activa (escenarios deportivos e infantiles), lo cual afecta la satisfacción de preferencias y características inherentes a los niños y personas que deseen recrearse activamente.
Asimismo, el porcentaje de presencia en los escenarios deportivos es mayor en las comunas dos, cuatro y nueve, con valores de 56,25\%, $70.00 \%$ y 54.55 $\%$ respectivamente; sin embargo, estos valores, al resultar bajos, son poco significativos, si se tiene en cuenta el total de elementos del espacio público distribuidos en estas zonas. La ciudad carece en su mayor parte del territorio de escenarios infantiles, tanto que el porcentaje mayor de presencia de estos elementos alcanzan un porcentaje máximo sobre el 50,00\% en las comunas dos, cuatro, cinco y siete, mientras el resto presen$\tan$ el $23,81 \%$ y el $35,48 \%$.

Montería sólo dispone de un elemento de espacio público regional (el Parque Lineal Ronda del Sinú), mientras existen 38 de escala zonal y 136 nivel vecinal, de tal modo que se requiere generar otros espacios públicos con mayor escala y diversidad.

Con relación a las condiciones espaciales, la carencia de espacio público en la ciudad, se sustenta en el alto valor que expresa el déficit cuantitativo promedio $(13,35 \mathrm{~m} 2 / \mathrm{hab}$., con relación al índice ideal de $15 \mathrm{~m} 2 /$ hab.), donde la comuna cinco (centro) presenta el menor déficit $(11,02 \mathrm{mt} 2 / \mathrm{hab}$.$) , lo cual puede asociar-$ se a la existencia del Parque Lineal Ronda del Sinú (escala regional). La escasez o nula presencia de espacio público efectivo en el resto de la ciudad, puede atribuirse, en parte, al crecimiento informal que ha tenido Montería, pues alrededor del $70 \%$ de sus barrios han surgido al margen de la ley, sin oferta 
de espacio público, vías, equipamientos necesarios para la satisfacción de las necesidades humanas individuales y colectivas, afectando una vez más, la calidad de vida urbana.

La distribución del espacio público se presenta de forma desigual e inequitativa en la ciudad de Montería, debido a que la población residente en cincuenta barrios accede óptimamente a espacios compuestos por los tres escenarios, mientras que 44 barrios de la ciudad no tienen acceso a ninguno de los elementos del espacio público de los 152 en total que existen. Algunos usuarios se desfavorecen más que otros, a causa de la falta de accesibilidad a elementos con escenarios que satisfagan sus gustos o intereses, como ocurre principalmente en áreas de las comunas tres, cuatro, cinco y seis, donde se evidencian las grandes brechas de desigualdad e inequidad social y la baja calidad de vida urbana, que existen en la ciudad de Montería.

\section{Literatura citada}

Alcaldía de Montería, Córdoba. (2002). Acuerdo 018 del 2002 mediante el cual se adopta el Plan de Ordenamiento Territorial del Municipio de Montería 20002011. Montería: Concejo Municipal de Montería.

Alcaldía de Montería, Córdoba. (2010). Acuerdo 029 del 2010 mediante el cual se adopta la Revisión y Ajuste del Plan de Ordenamiento Territorial del Municipio de Montería 2002-2015. Montería: Concejo Municipal de Montería.

Alcaldía Mayor de Bogotá y Programa de las Naciones Unidas. (2007).Ciudad y espacio público. En Alcaldía Mayor de Bogotá. Ciudad y espacio público el caso de los vendedores informales en el espacio público físico de Bogotá. (pp. 25-44). Bogotá: Alcaldía Mayor de Bogotá.

Alcaldía Mayor de Bogotá. (2008). Plan de Ordenamiento Territorial vigente de Bogotá. En Alcaldía Mayor de Bogotá. Título II Componente urbano, Subtítulo 3, Capítulo 10 Sistema de espacio público construido. Bogotá: Alcaldía Mayor de Bogotá. Recuperado de http://www.redcreacion. org/reddistrital/clasificacionparques.html

Borja, J. (1998). Ciudadanía y espacio público. Ambiente y desarrollo, XIV (3), 13-22.

Díaz, F. \& Ortiz, A. (2003). Ciudad e inmigración: uso y apropiación del espacio público en Barcelona. Barcelona. p. 399-407. Recuperado de http://descargas.cervantesvirtual.com/servlet/SirveObras/01371307433470683010035/020790.pdf?incr $=1$ 
Flores, R. \& González, M. (2007). Consideraciones sociales en el diseño y planificación de parques urbanos. Recuperado de http://www.cmq.edu.mx/documentos/Revista/revista24/est24_2.pdf

García, M. (2010). Narrativas que construyen ciudad: aportes de grupos de jóvenes a la convivencia en el espacio público de Bogotá. En P. Páramo \& M. García (Ed.). La dimensión social del espacio público aportes para la calidad de vida urbana. (pp. 41-77). Bogotá: Universidad Pedagógica Nacional y Universidad Santo Tomás de Aquino.

Giraldo, F. (2003).Espacio público y lugar. En F. Giraldo (Ed.). Ciudad y complejidad (pp.76-93). Bogotá: Fundación para la Investigación y la Cultura FICA.

Herrero, T., Pérez, E. \& Martín, J. (2001). La accesibilidad a zonas verdes urbanas mediante sistemas de información geográfica (SIG). Recuperado de http:// www.ingegraf.es/XVIII/PDF/Comunicacion 17054.pdf

Jiménez, J. (2009). Espacio público y calidad de vida urbana: análisis de las condiciones físico-espaciales en la ciudad de Montería. Tesis presentada para optar al título de pregrado en Geografía, no publicada. Universidad de Córdoba, Montería.

León, X. \& Naranjo, A. (2005). Quito: ¿Espacio público cada vez más privado? Quito-Ecuador. Recuperado de http://www.quitoparatodos.org/documentos/ publicaciones/qepcvmp.pdf

Negrete, V. (2001). El proceso de reubicación de población desplazada por la violencia en predios rurales del municipio de Montería. Recuperado de http:// www.disaster-info.net/desplazados/informes/acnur/reubicacion0creditos.htm

Páramo, P. (2010). El espacio público y la calidad de vida urbana. En P. Páramo \& M. García (Ed.). La dimensión social del espacio público aportes para la calidad de vida urbana. (pp. 15-26). Bogotá: Universidad Pedagógica Nacional y Universidad Santo Tomás de Aquino.

Pérez, E. (2004).Percepción del espacio público. Bitácora Urbano Territorial 1 (8), 27-31. Universidad Nacional de Colombia.

Presidencia de la República de Colombia. (1998). Decreto 1504 de 1998. Por el cual se reglamenta el manejo del espacio público en los planes de ordenamiento territorial. Bogotá: Ministerio de Desarrollo Económico. 
Scott, L. \& Marshall, A. (1998). Two Contrasting Approaches to Urban Accessibility for Individuals with Disabilities or Special Needs. The Journal of Urban Technology, 5 (1), 1-15.

United Nations Population Fund -UNFPA-. (2007). Estado de la población mundial. Liberar el potencial del crecimiento urbano. Capítulo 4. Recuperado de http://www.unfpa.org/swp/2007/spanish/ chapter_4/index.html

Yory, C. (2007). Espacio público y formación de ciudadanía. Bogotá: Universidad Pontificia Bolivariana.

Recepción: 31 de agosto de 2012

Evaluación: 13 de noviembre de 2012

Aprobación: 20 de febrero de 2013 